\title{
Analyse du discours et champ disciplinaire
}

Discourse Analysis and « disciplinary Field»

Dominique Maingueneau

\section{CpenEdition}

Journals

Édition électronique

URL : http://journals.openedition.org/questionsdecommunication/422

DOI : 10.4000/questionsdecommunication.422

ISSN : 2259-8901

\section{Éditeur}

Presses universitaires de Lorraine

\section{Édition imprimée}

Date de publication : 1 décembre 2010

Pagination : 185-196

ISBN : 978-2-8143-0056-9

ISSN : 1633-5961

\section{Référence électronique}

Dominique Maingueneau, " Analyse du discours et champ disciplinaire », Questions de communication [En ligne], 18| 2010, mis en ligne le 01 décembre 2012, consulté le 30 avril 2019. URL : http:// journals.openedition.org/questionsdecommunication/422 ; DOI : 10.4000/ questionsdecommunication.422 


\title{
ANALYSE DU DISCOURS ET CHAMP DISCIPLINAIRE
}

\begin{abstract}
Résumé. - Cet article commente un texte de Patrick Charaudeau précédemment paru dans Questions de communication: «Pour une interdisciplinarité «focalisée » dans les sciences humaines et sociales ». II ne s'agit pas d'une critique au sens étroit, car j'exprime mon accord avec les thèses défendues. Après en avoir résumé les idées essentielles, je discute certains points qui me semblent insuffisamment développés ou qui ne sont pas abordés par Patrick Charaudeau. En particulier, le statut singulier de l'analyse du discours, qui est interdisciplinaire par nature, et les problèmes que pose la définition de ce que Patrick Charaudeau appelle un « champ disciplinaire » (les sciences du langage ou les sciences de l'éducation par exemple); j'insiste sur la diversité de ces champs disciplinaires et sur leur caractère hétérogène. Cette discussion est illustrée par l'exemple des études littéraires.
\end{abstract}

Mots clés. - Charaudeau, analyse du discours, pluridisciplinarité, interdisciplinarité, discipline, champ disciplinaire. 
○ n m'a demandé de réagir à l'article de Patrick Charaudeau (20l0) publié par Questions de communication. Dans la mesure où je partage pour l'essentiel le point de vue qu'il défend et n'ai pas de programme alternatif à proposer, il m'est difficile de procéder à une critique tranchante. Plutôt que d'élaborer une contre-proposition, je vais pointer dans ce texte quelques éléments qui me semblent insuffisamment développés ou oubliés.

\section{Pluridisciplinarité, multidisciplinarité, interdisciplinarité}

L'article de Patrick Charaudeau se présente comme une sorte de manifeste dont le titre indique clairement le contenu : «Pour une interdisciplinarité «focalisée » dans les sciences humaines et sociales $\gg$. Le propos se veut donc ambitieux; il excède largement le cadre habituel des sciences du langage, domaine dans lequel travaille l'auteur. Patrick Charaudeau (2010 : 195) met le doigt là où ça fait mal, pour dire les choses trivialement, quand il part d'un paradoxe ainsi énoncé : « À une époque où il est beaucoup question de pluridisciplinarité ou d'interdisciplinarité [...] le système institutionnel et une certaine corporation de chercheurs ne prennent en considération que des études monodisciplinaires technicistes au nom de la scientificité ».

Comme on peut s'en douter, Patrick Charaudeau souhaite qu'il soit mis fin à ce décalage entre les bonnes intentions affichées et les pratiques effectives de nombreux chercheurs. L'argumentation qu'il développe à cette fin s'appuie sur ses propres recherches en communication, qui ont été menées dans une perspective d'analyse du discours où il place au centre le pouvoir d'influence qu'a le langage dans une situation de communication déterminée. Ainsi la réflexion épistémologique est-elle fondée sur l'expérience d'un chercheur qui revient sur les difficultés qu'il a rencontrées. Inutile de dire que les règles de bonne conduite qu'il propose fonctionnent également comme des justifications d'une entreprise qui a montré sa cohérence et sa fécondité, en particulier à travers les travaux du Centre d'analyse du discours de l'université Paris I3, dont il a été le principal animateur. Dans la mesure où l'analyse du discours comme champ spécifique n'a émergé qu'à la fin des années 60 , au moment où Patrick Charaudeau a commencé sa carrière d'enseignant-chercheur, ce retour sur sa propre pratique s'explique aussi par son statut de pionnier qui n'a pas pu s'appuyer sur un balisage préalable de son espace de recherche. II est de ceux qui ont été en quelque sorte obligés d'inventer une bonne part du chemin sur lequel ils s'avançaient, ce qui donne un prix particulier à cet article qui est à la fois un bilan et un programme. Pour lui, quand le chercheur en analyse du discours ne mène pas une réflexion sur sa pratique, il risque de basculer dans les généralités édifiantes mais largement stériles, ou à l'inverse dans les travaux d'orientation descriptive dénués de toute ambition. Contre ces dérives 
symétriques, son propos se veut normatif, et il est inévitable qu'il le soit. On ne peut pas se contenter de cartographier les travaux qui se réclament de l'analyse du discours, sans principe de hiérarchisation. Ce faisant, Patrick Charaudeau se distingue d'un grand nombre d'analystes du discours qui s'abritent derrière le présupposé rassurant que leurs démarches relèvent des « sciences du langage », évitant par là même de s'interroger plus avant sur ce qu'ils font. II se distingue aussi d'autres chercheurs, et tout particulièrement de ceux qui se situent hors des sciences du langage pour lesquelles l'analyse du discours serait seulement une « méthode qualitative » parmi d'autres, disponible dans la boîte à outils du chercheur en sciences humaines ou sociales. À la fin de son article, Patrick Charaudeau (2010:219) recense les trois écueils qu'à son sens il convient d'éviter: «Penser que seuls de grands systèmes explicatifs permettraient de rendre compte des phénomènes sociaux »; « penser que le recours simultané - et non critique - à plusieurs disciplines devrait permettre de mieux expliquer les phénomènes. Ce serait pratiquer un amalgame qui pourrait faire illusion mais ne serait que de la poudre aux yeux »; « défendre un relativisme neutre accumulant des études empiriques locales sans autre visée que d'apporter un petit caillou à l'édifice d'une cathédrale du savoir dont on ne verrait jamais que quelques pierres ».

La solution qu'il propose est de recourir à une véritable interdisciplinarité. Cette notion prêtant à équivoque, il procède à quelques distinctions terminologiques de base, qui convergent largement avec celles proposées il y a quelques années par Frédéric Darbellay (2005). En particulier celle entre la « pluridisciplinarité », qui ne serait qu'une juxtaposition de disciplines, et la «transdisciplinarité », à concevoir comme une traversée des disciplines qui aboutit à une « co-construction des savoirs » : pour Patrick Charaudeau ce serait,par exemple, le cas de Roland Barthes (" entre littérature, philosophie, psychanalyse »), de Michel Foucault (« entre philosophie et histoire ») ou d'Edgar Morin (« entre les différentes disciplines de la connaissance »). Chacun à sa façon a fait émerger « un discours sui generis construisant son propre lieu de pensée ». En revanche, dans la pluridisciplinarité chaque discipline garde son autonomie, ne réinterroge pas ses présupposés au regard de l'autre discipline, et ne fait qu'apporter (entreprise salutaire) son propre éclairage sur un objet d'étude lui-même analysé par d'autres. II s'agit d'une juxtaposition de points de vue qui délivrent chacun une connaissance particulière sur le phénomène étudié. Par exemple, à l'heure actuelle, le cNRS met en place des programmes de recherche associant des spécialistes des disciplines du « vivant » (biologie humaine ou animale, biodiversité, évolution, écologie) à des spécialistes des sciences humaines et sociales (sciences du langage, sciences de l'information et de la communication, sociologie). Les premiers, préoccupés par la façon dont les résultats de leurs travaux circulent dans l'espace public, cherchent à savoir comment les discours tenus par les scientifiques sont traités, transformés, voire dévoyés par les médias. Si ces scientifiques s'intéressent à ce que peut leur apporter d'autres disciplines, c'est de façon périphérique, dans la 
mesure où cela n'est pas destiné à remettre en cause leur propre discours en tant que scientifiques.

Quant à la « multidisciplinarité », Patrick Charaudeau y voit une autre forme de juxtaposition: on pourra également citer le cas de colloques qui convoquent plusieurs disciplines pour que chacune apporte son point de vue sur un même phénomène touchant la santé, la sécurité, l'environnement, etc. Dans ce cas, on parlera de multidisciplinarité que l'on distinguera de la pluridisciplinarité en ce que cette dernière est centrée sur un questionnement commun aux disciplines concernées, alors que la première est ouverte, sans limites, sur de grandes questions, sans problématisation particulière. À ces diverses stratégies de mises en relation des disciplines, l'auteur préfère l'interdisciplinarité qui consiste à établir de véritables connexions entre concepts, outils d'analyse et modes d'interprétation de différentes disciplines. II ne suffit pas d'ajouter des disciplines sur un même objet d'analyse; il faut faire se confronter diverses compétences disciplinaires afin de rendre plus pertinents ces concepts et outils d'analyse, ou d'étendre le champ des interprétations à partir de résultats eux-mêmes issus de protocoles d'analyse communs.

\section{Une conception trop optimiste?}

La position de Patrick Charaudeau sur les relations entre disciplines me paraît justifiée; ce n'est donc pas sur ce point qu'il me parait utile de revenir. En revanche, la condamnation des « grands systèmes explicatifs 》 serait sans doute à nuancer. Patrick Charaudeau (2010:219) illustre son propos en citant quelques « grands -ismes : les structuralisme, générativisme, interactionnisme ou historicisme d'hier, les sociologisme, économisme et juridisme d'aujourd'hui ». Recourir à de tels systèmes, « ce serait vouloir essentialiser des modèles d'analyse, au nom de la scientificité, et en les rendant dominants ouvrir la voie à un totalitarisme intellectuel » (ibid.). Certes, mais on peut se demander si cette liste ne mêle pas des phénomènes d'ordres distincts; en particulier, s'il ne faut pas distinguer deux sortes de « grands -ismes » : ceux qui sont de véritables systèmes explicatifs à visée totalisante et ceux qui sont plutôt ce qu'on appelle péjorativement des « modes intellectuelles ». Quoi que l'on fasse, on ne pourra pas empêcher qu'à un moment donné, le savoir ne soit sous l'emprise d'un certain nombre de leitmotive qui orientent les recherches et qui, par la suite, se révèleront pour une part porteurs d'illusions. Mais ces grands courants qu'on peut stigmatiser comme des « modes » sont également de puissants stimulants pour la recherche; ils permettent de définir de nouveaux programmes de travail, ils permettent aussi à de nouvelles générations de chercheurs de périmer des démarches antérieures et de faire valoir leur propre légitimité. Cela fait partie du fonctionnement normal de la recherche scientifique. Ainsi est-il inévitable qu'il y ait des secteurs plus « porteurs » que d'autres, voire parfois hégémoniques. 
Les hommes de science de la seconde moitié du XV\|le siècle considéraient la mécanique comme la discipline reine et ceux du XVIII siècle voyaient dans la loi de Newton le paradigme de la scientificité. Pour prendre un exemple plus proche de nous et qu'évoque Patrick Charaudeau : le Roland Barthes des années 50 ignore la linguistique et la psychanalyse, celui des années 60 la met au pinacle; le structuralisme est passé par là. Mais le Roland Barthes postérieur accompagne le reflux de ce même structuralisme, au point qu'aux États-Unis, on l'a intégré dans les penseurs « post-structuralistes ». Localement, tel spécialiste du discours peut bien décider d'entretenir des relations privilégiées avec la psychologie sociale, les mathématiques ou l'anthropologie de la connaissance, mais ces « contrats » d'échange locaux seront inévitablement influencés par les grands courants qui dans cette conjoncture dominent l'ensemble des sciences humaines et sociales. Pour prendre un exemple qui nous concerne plus particulièrement, Patrick Charaudeau et moi-même, en tant qu'analystes du discours, les problématiques sur le « discours » bénéficient aujourd'hui d'une aura évidente. II en va de même pour tout ce qui peut se réclamer de la « cognition ». Quand il énumère quelques « sous-disciplines » de la psychologie - « psychologie cognitive, sociale, clinique » (Charaudeau, $2010: 201$ ) -, il lui est difficile d'ignorer que l'une des trois, la psychologie cognitive, joue aujourd'hui un rôle dominant et exerce son influence bien au-delà de la psychologie. Bien entendu, un chercheur en analyse du discours ou en sociologie qui veut travailler avec des psychologues peut minorer la psychologie cognitive, mais s'il le fait, il aura de moins bonnes chances d'accéder à des financements et d'être reconnu au-delà de son domaine. L'interdisciplinarité au sens fort est une nécessité pour toute recherche scientifique, puisque toute vraie innovation dans ce domaine implique que les chercheurs sortent de leur espace, qu'ils entrent en dialogue avec d'autres modèles, d'autres disciplines, d'autres manières de penser. Mais cette nécessité peut aboutir à des résultats très variés, qui peuvent aller dans des directions opposées. On sait par exemple ce que la pensée de Ferdinand de Saussure doit à la théorie économique de son époque ou à la sociologie d'Émile Durkheim; pourtant, la démarche du Cours de linguistique générale va dans le sens d'une linguistique autonome, où le système est envisagé en lui-même et pour lui-même. De manière générale, il est très difficile d'énoncer des règles en matière de création intellectuelle. On est en droit de porter un jugement très critique sur la volonté de Pierre Bourdieu de placer la sociologie en position dominante, volonté qui l'a constamment conduit à polémiquer avec les tenants d'autres disciplines, reléguées dans la position subalterne de ceux qui agissent dans l'illusion. Le problème est de savoir si Pierre Bourdieu aurait pu produire ce qu'il a produit s'il n'avait été animé par ce désir de construire une science souveraine, s'il n'avait pas eu cette prétention de tout analyser à travers ses catégories : de la métaphysique de Martin Heidegger à la science en passant par la reproduction sociale, la différence sexuelle, la littérature ou la télévision. Certes, la confrontation avec d'autres disciplines est constante chez lui, mais elle tend à établir un rapport de forces au profit de sa propre entreprise, non un véritable dialogue. Les disciplines sont des réalités historiques qui ne se maintiennent que 
par les relations qu'elles entretiennent avec les zones du savoir qui les bornent. Autant dire que leur survie n'est jamais complètement assurée. Elles ne sortent pas nécessairement renforcées de toute mise en relation. On a beau encourager l'interdisciplinarité, on ne peut ignorer que l'interaction peut affaiblir l'un des termes de la relation. Pour prendre un exemple caricatural, si l'informatique entretient une relation privilégiée avec les mathématiques, cela ne manquera pas à la longue de transformer profondément les deux « partenaires » et d'amener certains mathématiciens à dénoncer une subordination de leur discipline à l'informatique. Si beaucoup de spécialistes de philosophie ou de littérature sont très réticents à l'égard de l'analyse du discours, c'est qu'une relation assidue avec cette dernière risque de délégitimer un certain nombre de pratiques en vigueur dans leurs champs, voire de faire disparaitre certains secteurs de ces champs. Autant dire que les résistances à l'interdisciplinarité font malheureusement partie du jeu, puisque l'interdisciplinarité n'est pas nécessairement une relation qui enrichit les deux partenaires.

\section{L'analyse du discours comme «sous-discipline»}

Un autre point qui me paraît insuffisamment discuté par Patrick Charaudeau concerne l'analyse du discours elle-même. Ce dernier lui donne le statut d'une « sous-discipline » à l'intérieur d'un « champ disciplinaire », celui des sciences du langage. Ainsi en est-il du champ des sciences psychologiques qui se subdivise en sous-disciplines (psychologie cognitive, psychologie sociale, psychologie clinique) mais qui se réfèrent toutes à une certaine conception du psychologique par opposition, par exemple, au sociologique ou à l'anthropologique. Ainsi en est-il du champ des sciences du langage qui se subdivise en diverses sous-disciplines (linguistique descriptive de la langue, linguistique cognitive, sociolinguistique, ethnolinguistique, linguistique du discours, etc.) mais qui se réfèrent toutes à des concepts communs de signe, forme et sens, ordres syntagmatique et paradigmatique, énoncé/énonciation et de procédures de recueil et analyse de corpus. II en est de même pour la sociologie, l'anthropologie et l'histoire parfois, où s'instaurent, à l'intérieur de chacune de ces sous-disciplines, des courants disciplinaires proposant des hypothèses, des catégories et une démarche d'analyse spécifiques. C'est le cas en sociologie avec les courants interactionniste, compréhensif, critique, dans le droit fil d'un constructivisme social (Corcuff, 1995). C'est également le cas en analyse du discours, sousdiscipline appartenant au champ des sciences du langage, à l'intérieur de laquelle on constate divers courants, les uns plutôt historicisants, d'autres idéologisants, d'autres plus formels, d'autres davantage communicationnels. Dans ce passage, on notera que c'est tantôt « l'analyse du discours », tantôt la « linguistique du discours » qui est présentée comme « sous-discipline des sciences du langage ». On peut penser que dans l'esprit de l'auteur l'analyse du discours est ellemême une composante de la « linguistique du discours », qui serait placée à 
un niveau supérieur. Mais c'est là un point de détail. L'un des problèmes que pose cette juxtaposition de « sous-disciplines » est qu'elle tend à atténuer leur hétérogénéité. Or, la question de l'interdisciplinarité ne se pose sans doute pas tout à fait de la même manière quand il s'agit de « linguistique descriptive de la langue » et d'analyse du discours. En effet, cette dernière se situe par nature aux frontières de multiples sciences humaines et sociales, ce qui n'est pas le cas de n'importe quelle sous-discipline des sciences du langage. Par exemple, la grammaire générative s'est constamment présentée comme une branche de la psychologie. II n'empêche que, pour la plupart de ses praticiens, elle est apparue avant tout comme une approche qui permettait de modéliser des propriétés du système linguistique mieux que ne pouvaient le faire d'autres courants linguistiques. Les considérations d'ordre psychologique jouaient finalement un rôle marginal dans cette affaire. Le grand scepticisme des linguistes français à l'égard de La linguistique cartésienne de Noam Chomsky (1966) n'a pas empêché le modèle génératif et transformationnel de connaître un très grand succès. Ce type de dissociation peut difficilement s'appliquer à l'analyse du discours qui, par nature, manipule des catégories hybrides, puisées à la fois dans la linguistique et dans diverses sciences humaines et sociales. Cela explique d'ailleurs en partie les difficultés que l'on rencontre quand on veut raconter les origines de l'analyse du discours: les nombreux auteurs qu'on peut identifier comme ayant joué un rôle dans sa fondation - si l'on peut parler ici de fondation - se trouvent placés dans des situations d'interdisciplinarité très diverses: Michel Pêcheux, par exemple, inscrit son entreprise d'analyse du discours entre la linguistique et une théorie de l'idéologie appuyée sur une conception althussérienne du matérialisme dialectique, Dell Hymes s'installe entre linguistique et anthropologie, etc. Et que dire d'auteurs comme Erving Goffman ou Harold Garfinkel qui situent leur démarche non dans l'orbite des sciences du langage mais dans celui de la sociologie? L'objet de l'analyse du discours n'est de toute façon qu'une zone de contact, une frontière, comme nous le rappelle ce passage célèbre de L'Archéologie du savoir de Michel Foucault (1969:65) : «Ce dont il s'agit ici n'est pas de neutraliser le discours, d'en faire le signe d'autre chose et d'en traverser l'épaisseur pour rejoindre ce qui demeure silencieusement en deçà de lui, c'est au contraire de le maintenir dans sa consistance, de le faire surgir dans la complexité qui lui est propre. En un mot, on veut, bel et bien, se passer des "choses". Les "dé-présentifier" ».

Ainsi, quand l'analyse du discours veut adopter une démarche foncièrement interdisciplinaire, peut-elle oublier qu'elle est elle-même prise dans une interdisciplinarité constitutive et que c'est précisément sur la manière de gérer cette interdisciplinarité constitutive que les grands courants d'analyse du discours divergent? Autant dire que même l'inscription de l'analyse du discours dans l'orbite des sciences du langage ne peut aller de soi. 


\section{Discipline et champ disciplinaire}

Venons-en à présent à la notion de « discipline ». Patrick Charaudeau souligne à juste titre que ce terme associe divers types de normes et de catégories et un appareil méthodologique. Une discipline est constituée d'un certain nombre de principes fondateurs, d'hypothèses générales, de concepts qui déterminent un champ d'étude et permettent en même temps de construire le phénomène en objet d'analyse. Se constitue ainsi un cadre conceptuel, et c'est à l'intérieur de celui-ci que peuvent être construites diverses théories, comme proposition d'une systémique autour de certaines catégories. Sans cadre théorique, point de discussion possible, car on ne saurait dire au nom de quoi on pourrait évaluer, confirmer ou contester les résultats d'une analyse. II s'agit là d'un principe de pertinence : discuter les explications que l'on donne sur le monde n'est possible que si l'on connaît le cadre conceptuel de référence, les catégories, les modes de raisonnement et les procédures d'analyse dont elles dépendent. Pour être opérationnel, ce cadre a besoin de se doter d'outils de description et de probation qui permettent à la fois de construire l'objet d'analyse, de le décomposer autant que de besoin, d'effectuer des distinctions ou des rapprochements, de mettre au jour des mécanismes de fonctionnement du phénomène étudié, et de présenter le tout sous forme de résultats à interpréter. Cet outillage constitue une méthodologie, et c'est ce couple théorie-méthodologie qui fonde une discipline en en déterminant le lieu de pertinence.

Patrick Charaudeau ne met pas ici l'accent sur la dimension foncièrement coopérative des disciplines, sur les communautés qu'elles rendent possibles et qui les rendent possibles, mais ce versant « sociologique » de la discipline est en filigrane dans la conception qu'il présente. Elle est tout à fait compatible avec d'autres caractérisations, qui soulignent plus fortement cet aspect « sociologique », comme celle de Jean-Marie Berthelot (1996: 99-100), pour qui les disciplines sont, à la fois,

« un lieu d'échange et de reconnaissance, et matrice de discours et de débats légitimes, [...] un lieu de ressources sociocognitives, de références autorisées, de normes partagées et d'exemples communs, permettant le tissage d'une tradition, problématique, conflictuelle, mais réelle, de connaissance. Cet espace de spécialisation disciplinaire est donc un lieu où peuvent s'articuler en une entreprise de connaissance légitime - non plus seulement socialement mais épistémologiquement, c'est-à-dire en une entreprise de connaissance argumentée - les divers langages par lesquels s'organise le travail analytique. Espace social de légitimation de savoirs, une discipline est, indissociablement, un espace logique de construction d'argumentations ».

S'il est indéniable que la discipline comporte un versant épistémologique, « heuristique », et un versant sociologique, il n'en est pas moins vrai que les relations entre ces deux versants sont très variables. II existe une tension permanente entre les critères heuristiques et les critères institutionnels dans le découpage des disciplines. Ce qui conduit à faire communément une distinction entre les disciplines au sens institutionnel, celles que reconnaissent les pratiques de l'administration universitaire, et les disciplines qui structurent la 
recherche, celles qui permettent aux acteurs des champs scientifiques d'élaborer leurs modélisations. Patrick Charaudeau ne traite pas de cette dimension institutionnelle; il faut dire qu'en général l'analyse du discours apparait surtout comme une discipline qui - du moins pour le moment - n'a guère d'existence institutionnelle forte. Et c'est particulièrement évident en France où les cadres disciplinaires structurent la gestion de la recherche, y compris les carrières des chercheurs. Même s'il ne le dit pas explicitement, il semble que, pour Patrick Charaudeau, la notion de discipline s'applique aux disciplines qui correspondent à des espaces de recherche consistants: la psychologie cognitive, et non l'ensemble du champ disciplinaire de la psychologie. Et on ne peut que lui donner raison sur ce point. Ces disciplines (qu'il appelle « sous-disciplines »), il les intègre dans des « champ disciplinaire » (par exemple les sciences du langage). II parle d'ailleurs de « champs disciplinaires » pour les sciences de l'information et de la communication ou pour les sciences de l'éducation, au même titre que pour les sciences du langage ou pour la psychologie. Selon lui, cette notion de champ disciplinaire est utile pour éviter certaines querelles qui ont lieu au sein des sciences humaines et sociales déniant aux sciences de l'éducation et aux sciences de l'information et de la communication le label de discipline. On a affaire à deux champs disciplinaires qui circonscrivent, chacun à sa façon, leur domaine d'étude, et qui, selon l'objet qu'ils étudient, peuvent avoir recours, autant que de besoin, à d'autres disciplines, telles la sociologie, la psychologie sociale, les linguistiques de la langue et du discours, l'histoire, etc. On peut se demander si cette notion de « champ disciplinaire » n'est pas trop laxiste, si par exemple les sciences de l'éducation sont un champ disciplinaire au même titre que les sciences du langage, telles que caractérisées par Patrick Charaudeau. Ainsi en est-il du champ des sciences du langage qui se subdivise en diverses sous-disciplines (linguistique descriptive de la langue, linguistique cognitive, sociolinguistique, ethnolinguistique, linguistique du discours) se référant toutes à des concepts communs de signe, forme et sens, ordres syntagmatique et paradigmatique, énoncé/énonciation et de procédures de recueil et analyse de corpus.

En fait, on peut très bien admettre que la notion de « champ disciplinaire » recouvre plusieurs régimes de disciplinarité. II est normal que l'existence d'un certain nombre d'espaces de recherche se justifie essentiellement en raison d'une forte demande sociale et qu'ils subissent immédiatement les effets des fluctuations de cette demande. Dans ce cas, le partage de « concepts » et de « procédures » communs à tous les membres d'un même champ disciplinaire passe au second plan. Pour ma part, j'aurais tendance à mettre davantage l'accent sur le caractère hétérogène de ce que Patrick Charaudeau appelle des « champs disciplinaires » et qu'on appelle communément « disciplines 》. En effet, ce qu'on entend par « sciences humaines et sociales » recouvre des réalités très diverses: il y a des disciplines qui répondent à des demandes sociales immédiates, d'autres qui mêlent recherche fondamentale et technè (ingénierie, gestion, médecine, etc.), d'autres qui sont obligées de privilégier la cohérence heuristique (sociologie, psychologie, linguistique), ce qui entraîne d'incessants débats épistémologiques. Et 
que dire de la philosophie, dont l'existence même est l'enjeu de chaque doctrine et pour laquelle l'idée même d'une appartenance aux « science humaine et sociale » est foncièrement inadéquate? Après tout, quand des chercheurs issus d'horizons très divers s'intéressent à un même domaine empirique, à un même « territoire », des espaces d'échanges se constituent qui peuvent avoir autant, voire davantage de productivité que des champs disciplinaires canoniques comme l'économie ou la psychologie. L'important - Patrick Charaudeau a raison de le souligner - est que les chercheurs ne se contentent pas de juxtaposer leurs démarches. Si on s'appuie sur une conception délibérément « sociocognitive » des disciplines de recherche (un certain nombre de gens qui partagent un certain nombre de références, publient dans les mêmes lieux, se lisent les uns les autres, se retrouvent aux mêmes colloques, etc.), et si l'on prend au sérieux l'exigence d'interdisciplinarité que défend vigoureusement Patrick Charaudeau, il me semble que la notion de champ disciplinaire perd un peu de son intérêt, en cela qu'elle ne peut pas être généralisée à l'ensemble des sciences humaines et sociales. Pour dire les choses simplement, je ne suis pas certain qu'il y ait davantage d'échanges à l'intérieur d'un même champ disciplinaire qu'entre tel ou tel secteur de champs qui sont supposés distincts. L'idée même que, dans le même champ disciplinaire, les chercheurs partageraient un certain nombre de présupposés et de méthodes est loin d'aller de soi.

Pour ne pas rester totalement dans le vague, on peut prendre l'exemple de l'étude de la littérature, dont l'assise académique est indiscutable. II ne s'agit pas d'une discipline de recherche mais, manifestement, d'un « champ disciplinaire », au sens où l'entend Patrick Charaudeau qui intégrerait diverses « sous-disciplines » : l'histoire littéraire, la narratologie, la stylistique, etc. Mais en fait, il apparaît bien difficile de découper l'ensemble de ce champ en disciplines; on y trouve aussi des divisions par courants (analyse thématique, psychanalytique, théorie de la réception, etc.), par périodes (ancien français, $\mathrm{XV} \|^{\mathrm{e}}$ siècle, etc.), par genres (roman, théâtre, etc.). Et que dire de la sociologie de la littérature, de l'histoire de l'édition, de la codicologie, de la littérature comparée, etc.? On aurait bien du mal à discerner des concepts ou des présupposés communs aux acteurs de ce champ. Certes, dans les années 60, s'est fait jour la prétention, rapportée aux formalistes russes, de donner une consistance théorique forte à l'étude de la littérature, mais l'acuité de ces interrogations s'est fortement émoussée avec le reflux du structuralisme. En fait, en France, c'est surtout l'existence d'un certain nombre d'institutions qui n'ont pas grand chose à voir avec la recherche (classes préparatoires, écoles normales supérieures, concours d'agrégation et de CAPES) et d'exercices (dissertation, explication de texte, etc.) qui assurent une certaine unité à cet espace. Sans cela, on aurait seulement affaire à un « territoire », à un groupement de chercheurs qui traitent du même objet. Mais ces derniers parlent-ils réellement du « même » objet, comme peuvent le faire des groupes qui travaillent sur l'internet ou la drogue? L'incertitude ne porte pas seulement sur le type de corpus à prendre en compte (œuvres véritables ou sous-littérature? anciennes ou contemporaines? française, européenne, 
universelle? etc.), mais aussi sur l'extension de ce domaine. Faut-il considérer seulement les textes et leurs abords immédiats, ou aller jusqu'au financement des théâtres, aux techniques de fabrication des livres, au statut juridique des écrivains, aux pratiques sociales attachées à la littérature (des visites de maisons d'écrivains aux reportages des magazines en passant par l'enseignement primaire et secondaire, les critiques de journaux, les images qui circulent dans la culture de masse, les prix, les bibliothèques, les loisirs, les adaptations d'œuvres au cinéma ou à la télévision, les traductions, etc.)? En d'autres termes, va-t-on prendre en compte un patrimoine d'œuvres consacrées ou le fait littéraire dans toute sa complexité? De la réponse à ce type de questions dépendent beaucoup de choses, en particulier les qualifications requises pour être un vrai « littéraire ». On peut même aller plus loin et douter de l'appartenance de ce champ disciplinaire aux sciences humaines et sociales. L'étude de la littérature est une activité qui mobilise deux populations très différentes. Pour les uns, la littérature se ramène à un thésaurus de grands textes à interpréter. Les autres se réclament des sciences humaines et sociales (sociologie de la littérature, analyse du discours littéraire par exemple) et ne font nullement du commentaire des « grands auteurs » le centre de leur activité. En outre, il existe un grand nombre d'universitaires qui se tiennent en-deçà de cette distinction entre deux populations, en particulier ceux qui se livrent à des travaux d'érudition ou d'établissement des textes et pour lesquels il est vain de se demander s'ils relèvent d'une approche herméneutique ou d'une approche en termes de sciences humaines et sociales. De toute façon, la divergence entre ces deux approches n'a rien de contingent. Dans la mesure où la littérature relève des « discours constituants » (Maingueneau, Cossutta, 1995), elle implique le partage des études littéraires entre ces deux paradigmes: l'un relevant des sciences humaines et sociales, l'autre d'une herméneutique des œuvres. Pour le premier, le discours littéraire ne bénéficie pas d'un régime d'extraterritorialité - ce qui ne veut pas dire qu'il n'a pas sa spécificité - mais se trouve intégré dans l'ensemble des pratiques d'une société. Pour le second, le « littéraire » a pour mission de faire vivre un thésaurus à travers lequel une communauté construit une identité. Mais ces deux paradigmes à l'intérieur du même « champ disciplinaire » n'ont rien d'étanche : les mêmes acteurs peuvent passer de l'un à l'autre et les tenants des approches herméneutiques empruntent et réorientent nombre d'éléments issus des sciences humaines et sociales. On le voit, quand on aborde ce champ, on ne trouve pas du tout les mêmes facteurs d'hétérogénéité qu'avec les sciences de l'éducation, la psychologie, l'économie, l'ingénierie, la philosophie, etc.

\section{Conclusion}

L'article de Patrick Charaudeau situe le débat à un niveau qui est indubitablement le plus intéressant pour la grande majorité des chercheurs en sciences humaines et sociales: celui des disciplines et des relations entre celles-ci. C'est là une 
position courageuse car on ne peut pas ignorer que c'est aussi un niveau où bien souvent les chercheurs préféreraient ne pas se poser trop de questions pour ne pas compromettre leur identité et leur légitimité. II est toujours plus facile de traiter de grands principes, ou de discuter de points très techniques qui n'engagent pas une interrogation sur les frontières. Le problème est que la recherche est enfermée dans un paradoxe qu'il lui faut gérer au mieux: d'un côté, les découpages disciplinaires semblent dérisoires, d'un autre, la recherche, comme réalité humaine, ne peut qu'être disciplinaire. C'est bien en focalisant l'attention sur l'interdisciplinarité et en assumant l'hétérogénéité des espaces dans lesquels s'effectue la recherche qu'on peut gérer cette difficulté de manière productive.

\section{Références}

Berthelot J.-M., 1996, Les Vertus de l'incertitude, Paris, Presses universitaires de France.

Charaudeau P., 20I0, « Pour une interdisciplinarité "focalisée" dans les sciences humaines et sociales », Questions de communications, I0, pp. 195-222.

Chomsky N., 1966, La linguistique cartésienne, un chapitre de I'histoire de la pensée rationaliste, suivi de la nature formelle du langage, trad. de l'anglais par N. Delanoë et D. Sperber, Paris, Éd. Le Seuil, 1969.

Darbellay F., 2005, Interdisciplinarité et transdisciplinarité en analyse des discours. Complexité des textes, intertextualité et transtextualité, Genève, Slatkine.

Foucault M., 1969, L'Archéologie du savoir, Paris, Gallimard.

Maingueneau D, Cossutta F., 1995, «L'analyse des discours constituants », Langages, I 17 , pp. I I2-125. 